OPEN ACCESS

Edited by:

Alfredo Ercoli,

University of Messina, Italy

Reviewed by:

Giacomo Corrado,

Agostino Gemelli University

Polyclinic, Italy

Stefano Restaino,

Ospedale Santa Maria della

Misericordia di Udine, Italy

${ }^{*}$ Correspondence:

Ping Liu

lpivy@126.com

Chunlin Chen

cc11@smu.edu.cn

orcid.org/0000-0002-1708-3047

tThese authors have contributed equally to this work

Specialty section: This article was submitted to

Surgical Oncology,

a section of the journal

Frontiers in Surgery

Received: 07 April 2021

Accepted: 06 August 2021

Published: 07 September 2021

Citation:

Li P, Liu J, Wang L, Kang S, Yang Y, Guo J, Yao J, Lu A, Wang Z, Lin B,

Li Z, Bin X, Lang J, Liu P and Chen C

(2021) The Effect of Laparoscopic

Radical Hysterectomy Surgical

Volume on Oncology Outcomes in

Early-Stage Cervical Cancer.

Front. Surg. 8:692163.

doi: 10.3389/fsurg.2021.692163

\section{The Effect of Laparoscopic Radical Hysterectomy Surgical Volume on Oncology Outcomes in Early-Stage Cervical Cancer}

Pengfei $\mathrm{Li}^{1+}$, Jiaqi Liu ${ }^{1 \dagger}$, Li Wang ${ }^{2 \dagger}$, Shang Kang ${ }^{3}$, Ying Yang ${ }^{4}$, Jianxin Guo ${ }^{5}$, Jilong Yao ${ }^{6}$, Anwei Lu ${ }^{7}$, Zhonghai Wang ${ }^{8}$, Bin Lin ${ }^{9}$, Zhiqiang $\mathrm{Li}^{1}$, Xiaonong Bin ${ }^{10}$, Jinghe Lang ${ }^{1,11}$, Ping Liu ${ }^{1 *}$ and Chunlin Chen ${ }^{1 *}$

${ }^{1}$ Department of Obstetrics and Gynecology, Nanfang Hospital, Southern Medical University, Guangzhou, China, ${ }^{2}$ Department of Gynecologic Oncology, Affiliated Cancer Hospital of Zhengzhou University, Zhengzhou, China, ${ }^{3}$ Department of Gynecology, Fourth Hospital of Hebei Medical University, Shijiazhuang, China, ${ }^{4}$ Department of Obstetrics and Gynecology, Xinqiao Hospital, Army Medical University, Chongqing, China, ${ }^{5}$ Department of Obstetrics and Gynecology, Daping Hospital, Army Medical University, Chonqing, China, ${ }^{6}$ Department of Gynecology, Shenzhen Maternity and Child Healthcare Hospital, Shenzhen, China, ' Department of Gynecology, Shenzhen Hospital of Southern Medical University, Shenzhen, China, ${ }^{8}$ Department of Gynecology, Shenzhen Sixth People's Hospital, Shenzhen, China, ${ }^{9}$ Department of Obstetrics and Gynecology, China-Japan Friendship Hospital, Beijing, China, ${ }^{10}$ Department of Epidemiology, College of Public Health, Guangzhou Medical University, Guangzhou, China, ${ }^{11}$ Department of Obstetrics and Gynecology, Peking Union Medical College Hospital, Peking Union Medical College, Beijing, China

Purpose: To examine the association between surgical volume and surgical and oncological outcomes of women with stage IB1 cervical cancer who underwent laparoscopic radical hysterectomy $(\mathrm{LRH})$.

Methods: We retrospectively analyzed the oncological outcomes of 1,137 patients with stage IB1 cervical cancer receiving LRH from 2004 to 2016. The surgical volume for each surgeon was defined as low [fewer than 50 surgeries, $n=392(34.5 \%)$ ], mid [51-100 surgeries, $n=315(27.7 \%)]$, and high [100 surgeries or more, $n=430(37.8 \%)]$. Surgical volume-specific survival was examined with Kaplan-Meier analysis, multivariable analysis, and propensity score matching.

Results: The operative times of the high-volume group (227.35 $\pm 7.796 \mathrm{~min})$ were significantly shorter than that of the low- $(272.77 \pm 4.887 \mathrm{~min}, p<0.001)$ and mid-volume $(255.86 \pm 4.981 \mathrm{~min}, p<0.001)$ groups. Blood loss in the high-volume group $(169.42 \pm 8.714 \mathrm{ml}$ ) was significantly less than that in the low-volume group (219.24 $\pm 11.299 \mathrm{ml}, p=0.003$ ). The 5-year disease-free survival (DFS) and overall survival (OS) in the low-volume, mid-volume, and high-volume groups were similar (DFS: 91.9, 86.7, and 89.2\%, $p=0.102$; OS: 96.4, 93.5, and 94.2\%, $p=0.192$ ). Multivariable analysis revealed surgical volume was not an independent risk factor for OS or DFS. The rate of intraoperative and postoperative complications was similar among the three groups $(p=0.210)$.

Conclusions: Surgical volume of LRH may not be a prognostic factor for patients with stage IB1 cervical cancer. Surgery at high-volume surgeon is associated with decreased operative time and blood loss.

Keywords: cervical cancer, laparoscopy, IB1 stage, surgical volume, oncology outcome 


\section{INTRODUCTION}

Radical hysterectomy (RH) with pelvic lymphadenectomy is the conventional treatment for early-stage cervical cancer (1). The laparoscopic technique was first reported for RH in 1992 (2), and since then, minimally invasive surgery has become a common surgical approach because of the advantages of less intraoperative blood loss, fewer operative complications, and shorter hospital stay when compared with traditional open surgery (3-7). However, the laparoscopic approach to cervical cancer (LACC) trial (8), a high-quality international multicenter randomized controlled trial, reported that laparoscopic radical hysterectomy (LRH) was closely related to worse oncology outcomes compared with abdominal radical hysterectomy (ARH). Consequently, the National Comprehensive Cancer Network (NCCN) guidelines recommend laparotomy as the standard approach for a $\mathrm{RH}$ for cervical cancer (9).

Several recent retrospective studies have also demonstrated that minimally invasive $\mathrm{RH}$ was associated with shorter OS and DFS rates than that of ARH (9-12). But these studies failed to analyze the reasons. The surgical experience of surgeons is often considered to affect the outcomes of surgery. So, the surgical experience of surgeons may be one of the reasons influencing the oncology outcomes of LRH (1315). However, there are limited data specifically analyzing the impact of the experience of surgeons in LRH, and most of their main concern was surgical skills or shortterm effects (16-18), and little concentration was focused on long-term survival outcomes. Most of these studies involved single centers and surgeons, with few cases included (17, 19, 20).

Therefore, based on the large clinical diagnosis and treatment database of cervical cancer in the mainland China (Four C) database, we explored the association between the LRH volume of surgeons and long-term oncological outcomes of women with early-stage cervical cancer who underwent LRH. We divided the patients into low-, medium-, and high-surgical volume groups according to the surgical experience of surgeons and compared the 5-year overall survival rates and disease-free survival rates of patients with stage IB1 cervical cancer among the three groups.

\section{METHODS}

\section{Data Source}

The data of this study derive from the clinical diagnosis and treatment for cervical cancer in mainland China (Four C) database, a cervical cancer-specialized disease database $(n=$ $46,313)$ that covers consecutive patients with cervical cancer in 37 hospitals in mainland China treated between January 2004 and December 2016. The Ethics Committee of Nanfang Hospital, Southern Medical University, approved this retrospective study (ethics number NFEC-2017-135). Written informed consent was waived by the ethics committee, as the information of human medical documents was retrospectively gathered and analyzed and patient data were unidentifiable in this study. The identifier of the clinical trial is CHiCTR1800017778 (International
Clinical Trials Registry Platform Search Port, http://apps.who. int/trialsearch/).

Using standardized data collection and quality control procedures, trained gynecological oncology staff collected the clinical data from patient files and the medical record management system in the hospitals. The details of the data sources and methods were the same as those previously reported $(21,22)$. For patients undergoing surgical treatment, the collected data, including demographic details, preoperative examination results, surgical information, pathological results, preoperative and postoperative adjuvant treatment details, complications, hospitalization time, expenses, and follow-up, contained almost all the information during the treatment of cervical cancer. In this database, the International Federation of Gynecology and Obstetrics (FIGO) stage was recorded and corrected by tumor size according to the FIGO 2009 staging system. Tumor size was evaluated by pathological records. To ensure the accuracy of the collected data, two uniformly trained staff members used EpiData software (EpiData Association, Odense M, Denmark) to input and proofread the same data from each hospital.

All follow-up procedures were carried out by trained gynecological oncology staff at each center to keep the personal data of patients confidential and to provide disease-management guidance. The follow-up information, including survival status, time of death, recurrence time, recurrence site, and treatment after recurrence, was gathered through the return visit system or telephone follow-up. Oncological outcomes were assessed according to the recorded information, and the last day of the return visit or telephone follow-up was defined as the last followup. The follow-up rate of oncological outcomes in this database is $72.7 \%$.

\section{Inclusion and Exclusion Criteria}

The inclusion criteria were as follows: (1) age $>18$ years old; (2) FIGO 2009 stage IB1; (3) squamous cell carcinoma, adenocarcinoma, or adenosquamous cell carcinoma; (4) Q-M type $\mathrm{B} 2$ or type $\mathrm{C} 2$ hysterectomy + pelvic lymphadenectomy; (5) available survival outcomes; (6) no preoperative treatments; and (7) standard postoperative adjuvant treatment after the operation.

The exclusion criteria were as follows: (1) pregnancy; (2) cervical stump cancer; (3) combined with other malignant tumors; and (4) history of pelvic surgery.

\section{Definitions and Outcome Measures}

In this study, patients treated by surgeons with different LRH experiences were divided into three groups (19): low-volume surgeons $(<51)$, mid-volume surgeons (51-100), and highvolume surgeons $(>100)$. Outcome measures included operative technique (operative time and blood loss) and oncological outcome (5-year OS and DFS).

The OS was defined as the time from the date of surgery to the date of death from any cause. DFS was defined as the time from the date of surgery to the date of disease recurrence or death from cervical cancer, and patients with no evidence of recurrence or death were censored at the date of the last follow-up or return visit. 


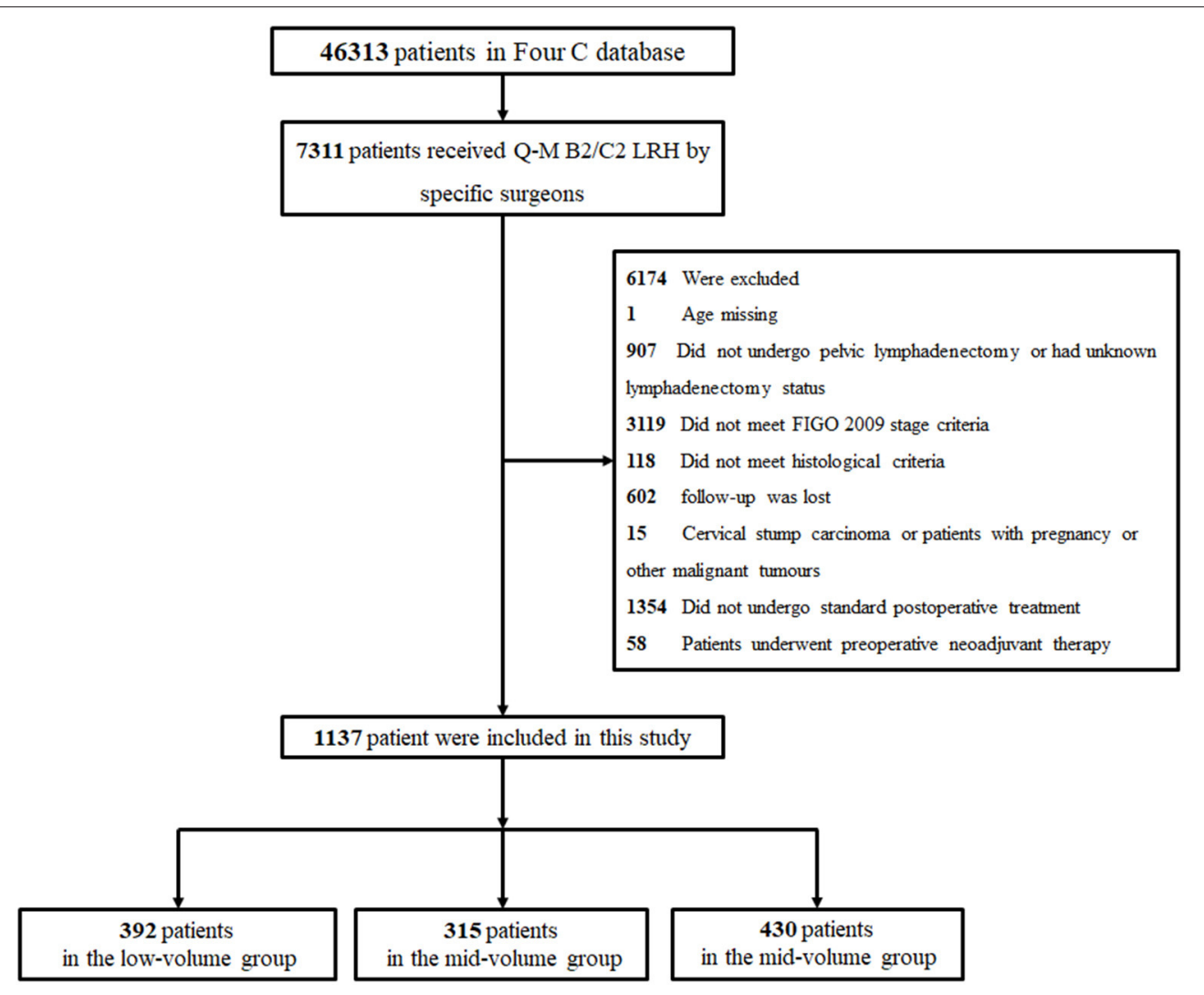

FIGURE 1 | Flowchart of patients included in the analysis.

According to FIGO guidelines (23), standard postoperative treatment was defined by any one pathological high-risk factor (positive para-aortic or pelvic nodes, parametrial extension, and positive margins) or any two or more pathological medium-risk factors (tumor size $>4 \mathrm{~cm}$, lymph-vascular space invasion (LVSI), stromal invasion), who received radiotherapy or radiochemotherapy or those with no postoperative high-risk factor and one or more medium-risk factors, who did not receive postoperative radiotherapy or radiochemotherapy.

Complications were classified into intraoperative complications and postoperative complications. Intraoperative complications included ureteral injury, bladder injury, bowel injury, vascular injury, and obturator nerve injury. Postoperative complications within 2 years of surgery included bowel obstruction, pelvic hematoma, hemorrhage, vesicovaginal fistula, ureterovaginal fistula, ureteral fistula, rectovaginal fistula, venous thromboembolism, and chylous leakage (24).

\section{Statistical Methods}

All statistical procedures were processed with Statistical Product and Service Solutions (SPSS) 23.0 statistical software (SPSS, Inc., Chicago, IL, USA). Between-group differences in the baseline characteristics were assessed through independent two-sample $t$ tests or Pearson's chi-squared test. Quantitative data are shown as the mean $\pm \mathrm{SD}(\mathrm{x} \pm \mathrm{s})$, and nominal-scale data are shown as percentages (\%). The 5-year OS and DFS rates of LRH and ARH were calculated and compared using the Kaplan-Meier curve and the log-rank test. A Cox proportional hazards model was used to estimate hazard ratio (HR)s and 95\% CIs for the effect of treatment on 5-year OS and DFS; known factors that may affect the oncological outcome of cervical cancer were included in this multivariate model to adjust for case-mix, including age, hospital type, region, city class, finance, year, Q-M type, histology, LVSI, stromal invasion, uterine corpus invasion, parametrial tumor involvement, surgical margin invasion, lymph node metastasis, preoperative treatment condition, and postoperative adjuvant treatment. Propensity score matching (PSM, 1:1) was used to balance differences in the data analysis; the variables included were the same as those above. A $P$-value $<0.05$ from two-sided tests was regarded as significant.

\section{RESULTS}

Among the 46,313 patients described in the database, 1,137 were included in this study (392 patients in the low-volume group, 315 patients in the mid-volume group, and 430 patients in the highvolume group). The flow diagram of recruitment and exclusion is illustrated in Figure 1. The median follow-up time was 42 months. The clinicopathologic characteristics of the three groups are provided in Table 1 . The high-volume group comprised more 
TABLE 1 | Clinical and pathological characteristics of the three groups.

\begin{tabular}{|c|c|c|c|c|c|c|c|}
\hline & \multicolumn{2}{|c|}{ Low } & \multicolumn{2}{|c|}{ Mid } & \multicolumn{2}{|c|}{ High } & \multirow[t]{2}{*}{$P$} \\
\hline & \multicolumn{2}{|c|}{$(n=392, \%)$} & \multicolumn{2}{|c|}{$(n=315, \%)$} & \multicolumn{2}{|c|}{$(n=430, \%)$} & \\
\hline Age (y) & \multicolumn{2}{|c|}{$46.68 \pm 9.175$} & \multicolumn{2}{|c|}{$47.35 \pm 9.281$} & \multicolumn{2}{|c|}{$47.25 \pm 9.312$} & 0.569 \\
\hline Hospital type & & & & & & & 0.010 \\
\hline General hospital & 210 & $53.60 \%$ & 201 & $63.80 \%$ & 220 & $51.20 \%$ & \\
\hline Tumor Hospital & 138 & $35.20 \%$ & 91 & $28.90 \%$ & 162 & $37.70 \%$ & \\
\hline Women-children specialty hospital & 44 & $11.20 \%$ & 23 & $7.30 \%$ & 48 & $11.20 \%$ & \\
\hline Region & & & & & & & $<0.001$ \\
\hline North China & 116 & $29.60 \%$ & 82 & $26.00 \%$ & 207 & $48.10 \%$ & \\
\hline South China & 141 & $36.00 \%$ & 123 & $39.00 \%$ & 58 & $13.50 \%$ & \\
\hline Central China & 42 & $10.70 \%$ & 17 & $5.40 \%$ & 26 & $6.00 \%$ & \\
\hline East China & 88 & $22.40 \%$ & 58 & $18.40 \%$ & 32 & $7.40 \%$ & \\
\hline Southwest China & 5 & $1.30 \%$ & 35 & $11.10 \%$ & 107 & $24.90 \%$ & \\
\hline City class & & & & & & & $<0.001$ \\
\hline First-tier & 140 & $35.70 \%$ & 65 & $20.60 \%$ & 21 & $4.90 \%$ & \\
\hline Second-tier & 176 & $44.90 \%$ & 152 & $48.30 \%$ & 318 & $74.00 \%$ & \\
\hline Third-tier and above & 76 & $19.40 \%$ & 98 & $31.10 \%$ & 91 & $21.20 \%$ & \\
\hline Economics & & & & & & & 0.018 \\
\hline Countryside & 205 & $52.30 \%$ & 191 & $60.60 \%$ & 246 & $57.20 \%$ & \\
\hline Urban & 141 & $36.00 \%$ & 107 & $34.00 \%$ & 153 & $35.60 \%$ & \\
\hline Unknown & 46 & $11.70 \%$ & 17 & $5.40 \%$ & 31 & $7.20 \%$ & \\
\hline Year & & & & & & & 0.036 \\
\hline $2005-2013$ & 117 & $29.80 \%$ & 83 & $26.30 \%$ & 154 & $35.80 \%$ & \\
\hline 2014-2015 & 149 & $38.00 \%$ & 134 & $42.50 \%$ & 167 & $38.80 \%$ & \\
\hline 2016 & 126 & $32.10 \%$ & 98 & $31.10 \%$ & 109 & $25.30 \%$ & \\
\hline Q-M Type & & & & & & & $<0.001$ \\
\hline QM-B2 & 251 & $64.00 \%$ & 101 & $32.10 \%$ & 43 & $10.00 \%$ & \\
\hline QM-C2 & 141 & $36.00 \%$ & 214 & $67.90 \%$ & 387 & $90.00 \%$ & \\
\hline Number of lymph nodes removed & $21.97 \pm 9.917$ & $23.16 \pm 10.934$ & $21.42 \pm 11.596$ & 0.096 & & & \\
\hline Histology & & & & & & & 0.108 \\
\hline Squamous & 338 & $86.20 \%$ & 262 & $83.20 \%$ & 384 & $89.30 \%$ & \\
\hline Adenocarcinoma & 51 & $13.00 \%$ & 49 & $15.60 \%$ & 40 & $9.30 \%$ & \\
\hline Adenosquamous & 3 & $0.80 \%$ & 4 & $1.30 \%$ & 6 & $1.40 \%$ & \\
\hline LVSI & & & & & & & 0.026 \\
\hline No & 346 & $88.30 \%$ & 258 & $81.90 \%$ & 354 & $82.30 \%$ & \\
\hline Yes & 46 & $11.70 \%$ & 57 & $18.10 \%$ & 76 & $17.70 \%$ & \\
\hline Deep stromal invasion & & & & & & & 0.008 \\
\hline No & 250 & $63.80 \%$ & 179 & $56.80 \%$ & 276 & $64.20 \%$ & \\
\hline Yes & 81 & $20.70 \%$ & 95 & $30.20 \%$ & 112 & $26.00 \%$ & \\
\hline Unreported & 61 & $15.60 \%$ & 41 & $13.00 \%$ & 42 & $9.80 \%$ & \\
\hline Uterine infiltration & & & & & & & 0.129 \\
\hline No & 380 & $96.90 \%$ & 297 & $94.30 \%$ & 405 & $94.20 \%$ & \\
\hline Yes & 12 & $3.10 \%$ & 18 & $5.70 \%$ & 25 & $5.80 \%$ & \\
\hline Parametrial involvement & & & & & & & 0.257 \\
\hline No & 389 & $99.20 \%$ & 310 & $98.40 \%$ & 428 & $99.50 \%$ & \\
\hline Yes & 3 & $0.80 \%$ & 5 & $1.60 \%$ & 2 & $0.50 \%$ & \\
\hline Surgical margin invasion & & & & & & & 0.471 \\
\hline No & 389 & $99.20 \%$ & 311 & $98.70 \%$ & 428 & $99.50 \%$ & \\
\hline Yes & 3 & $0.80 \%$ & 4 & $1.30 \%$ & 2 & $0.50 \%$ & \\
\hline Lymph node metastasis & & & & & & & 0.196 \\
\hline No & 350 & $89.30 \%$ & 276 & $87.60 \%$ & 366 & $85.10 \%$ & \\
\hline Yes & 42 & $10.70 \%$ & 39 & $12.40 \%$ & 64 & $14.90 \%$ & \\
\hline Adjuvant therapy & & & & & & & 0.157 \\
\hline None & 327 & $83.40 \%$ & 244 & $77.50 \%$ & 332 & $77.20 \%$ & \\
\hline Chemotherapy alone & 0 & $0.00 \%$ & 0 & $0.00 \%$ & 0 & $0.00 \%$ & \\
\hline Radiotherapy alone & 4 & $1.00 \%$ & 6 & $1.90 \%$ & 10 & $2.30 \%$ & \\
\hline Chemoradiotherapy & 61 & $15.60 \%$ & 65 & $20.60 \%$ & 88 & $20.50 \%$ & \\
\hline
\end{tabular}


patients from the north and southwest region and second-tier cities $(p<0.001)$ and less patients from the general hospital $(p=$ $0.010)$ than the other groups. The low-volume group comprised less patients from the countryside than the other groups $(p=$ 0.018 ). Women in the high-volume group were more likely to receive Querleu-Morrow (Q-M) type C2 RH compared with the other two groups $(p<0.001)$. The patients in the high-volume group were more likely to have LVSI $(p=0.026)$ compared with the women in the other two groups; those in the mid- and high-volume groups were more likely to have deep stromal than were those in the low-volume group $(p=0.008)$. No significant differences were found among the three groups in the number of lymph nodes removed, histological types, uterine infiltration, parametrial involvement, surgical margin invasion, lymph node metastasis, and postoperative treatment $(p>0.05)$.

\section{Surgical Outcomes}

The operative times in low-, mid-, and high-volume groups were $272.77 \pm 4.887 \mathrm{~min}, 255.86 \pm 4.981 \mathrm{~min}$, and $227.35 \pm 7.796 \mathrm{~min}$, respectively $(p<0.001)$. There was no significant difference in operative time between the low- and mid-volume groups ( $p=$ 0.096). While the operative times of the high-volume group were significantly shorter than the low- and mid-volume groups (all $p$ $<0.001$ ). Among the 1,137 patients, the average intraoperative blood loss was $219.24 \pm 11.299 \mathrm{ml}, 198.08 \pm 10.595 \mathrm{ml}$, and $169.42 \pm 8.714 \mathrm{ml}$ in the low-, mid-, and high-volume groups, respectively $(p=0.002)$. There was no significant difference between the mid-volume group and the low- $(p=0.258)$ and high-volume groups $(p=0.058)$, but blood loss in the highvolume group was significantly less than that in the low-volume group $(p=0.003)$.

\section{Results of Complications}

In the total study population, complications occurred in $18(4.6 \%), 14(4.4 \%)$, and $30(7.0 \%)$ patients in the low-, mid-, and high-volume group, respectively. The complication data are shown in Table 2. The rate of any one complication refers to the incidence of one or more complications in a patient, which had similar injury rates among the three groups $(p=0.210)$; moreover, the three groups had similar rates of intraoperative $(p$ $=0.745)$ and postoperative $(p=0.145)$ complications.

Among the intraoperative complications, the main complication was ureteral injury $(9 / 1137,0.79 \%)$. The rate of ureteral injury was higher in the high-volume group than in the low- and mid-volume groups, but the difference among them was insignificant ( $p=0.317)$; the obturator nerve and bladder injury rates were similar among the three groups $(p>$ 0.050 ). There were no cases of vascular injury or bowel injury in either group.

Among the postoperative complications, the main complications were ureterovaginal fistula (16/1137, 1.41\%), venous thromboembolism (14/1137, 1.23\%), and bowel obstruction $(7 / 1137,0.61 \%)$. The three groups had similar rates of vesicovaginal fistula, ureterovaginal fistula, rectovaginal fistula, venous thromboembolism, bowel obstruction, chylous leakage, pelvic hematoma, and active abdominal and pelvic bleeding $(p>0.05)$.

\section{Oncological Outcomes}

In 1,137 cases, 35 cases died and 83 cases recurred. In the low, mid-, and high-volume groups, the 5-year DFS was 91.9, 86.7, and $89.2 \%$; the 5 -year OS was $96.4,93.5$, and $94.2 \%$; there was no significant difference between the three groups (DFS: $p=0.102$; OS: $p=0.192$ ). The Kaplan-Meier survival curves are shown in Figure 2. In the multivariable analysis, adjusting for the casemix, patients in different volume groups were not an independent worse prognostic factor for a worse 5-year DFS or OS (DFS: $p=$ 0.239; OS: $p=0.206$, Table 3).

After 1:1 PSM, the clinicopathological characteristics were well-balanced (Table 4). For the low-volume group vs. the midvolume group, including 201 patients in each, there was no significant difference in the 5-year DFS or OS (DFS: 91.0 vs. $85.8 \%, p=0.192$; OS: 95.2 vs. $93.1 \%$; $p=0.076$ ). The KaplanMeier survival curves are shown in Figure 3. In Cox regression analysis adjusting for the above factors, the volume of surgery was not an independent risk factor for DFS and OS (DFS: $p=0.120$; OS: $p=0.051$ ).

There were also no significant differences in OS and DFS between the low-volume and high-volume groups, including 145 patients in each (DFS: 95.5 vs. $94.2 \%, p=0.870$; OS: 94.9 vs. $96.3 \% ; p=0.452$ ). The Kaplan-Meier survival curves are shown in Figure 3. After eliminating the case-mix factors by Cox proportional hazards model analysis, the volume of surgery was not an independent risk factor for DFS and OS (DFS: $p=0.426$; OS: $p=0.839$ ).

Between the mid-volume and high-volume groups, with 202 patients in each group, there were no significant differences in DFS and OS (DFS: 88.5 vs. $83.1 \%, p=0.709$; OS: 93.6 vs. $92.5 \% ; p=0.797)$. The Kaplan-Meier survival curves are shown in Figure 3. After eliminating the case-mix factors by Cox regression analysis, the surgical volume of surgeons was not an independent risk factor for DFS and OS (DFS: $p=0.477$; OS: $p$ $=0.426)$.

\section{DISCUSSIONS}

In this study, we divided patients into low-, mid-, and highvolume groups according to the total volume of LRH experience of surgeons and analyzed their effects on long-term oncological outcomes (DFS and OS), surgical outcomes (operative time and blood loss), and operative complications through a multisurgeon, large sample, and multicenter study. Key findings are that the three groups showed similar 5-year DFS and OS rates in the unadjusted and adjusted analysis, which means that the surgical volume of surgeons of LRH may not affect the long-term survival outcomes for patients with stage IB1 cervical cancer. We also found that intraoperative blood loss decreased and operative time was significantly shortened with an increase in surgical volume, while the incidence rate of operative complications among the three groups was similar.

Following the LACC trial, several retrospective studies have also demonstrated that minimally invasive $\mathrm{RH}$ was associated with worse oncological outcomes than that of ARH. There have been several changes to the recommendations for LRH in 
TABLE 2 | Intraoperative and postoperative complications among low-, mid-, and high-volume group.

\begin{tabular}{|c|c|c|c|c|c|c|c|}
\hline & \multicolumn{2}{|c|}{ Low } & \multicolumn{2}{|c|}{ Mid } & \multicolumn{2}{|c|}{ High } & \multirow[t]{2}{*}{$P$} \\
\hline & $n$ & $\%$ & $N$ & $\%$ & $n$ & $\%$ & \\
\hline Any 1 complication & 18 & $4.60 \%$ & 14 & $4.40 \%$ & 30 & $7.00 \%$ & 0.210 \\
\hline Intraoperative complication & 4 & $1.00 \%$ & 4 & $1.30 \%$ & 7 & $1.60 \%$ & 0.745 \\
\hline Vascular injury & 0 & $0.00 \%$ & 0 & $0.00 \%$ & 0 & $0.00 \%$ & - \\
\hline Ureteral injury & 1 & $0.30 \%$ & 3 & $1.00 \%$ & 5 & $1.20 \%$ & 0.317 \\
\hline Obturator nerve injury & 1 & $0.30 \%$ & 0 & $0.00 \%$ & 0 & $0.00 \%$ & 0.386 \\
\hline Bladder injury & 2 & $0.50 \%$ & 1 & $0.30 \%$ & 1 & $0.20 \%$ & 0.792 \\
\hline Bowel injury & 0 & $0.00 \%$ & 0 & $0.00 \%$ & 0 & $0.00 \%$ & - \\
\hline Postoperative complication & 14 & $3.60 \%$ & 10 & $3.20 \%$ & 25 & $5.80 \%$ & 0.145 \\
\hline Vesicovaginal fistula & 3 & $0.80 \%$ & 1 & $0.30 \%$ & 1 & $0.20 \%$ & 0.478 \\
\hline Ureterovaginal fistula & 6 & $1.50 \%$ & 4 & $1.30 \%$ & 6 & $1.40 \%$ & 0.958 \\
\hline Rectovaginal fistula & 0 & $0.00 \%$ & 0 & $0.00 \%$ & 3 & $0.70 \%$ & 0.084 \\
\hline Venous thromboembolism & 1 & $0.30 \%$ & 4 & $1.30 \%$ & 9 & $2.10 \%$ & 0.058 \\
\hline Bowel obstruction & 2 & $0.50 \%$ & 2 & $0.60 \%$ & 3 & $0.70 \%$ & 0.942 \\
\hline Chylous leakage & 1 & $0.30 \%$ & 0 & $0.00 \%$ & 2 & $0.50 \%$ & 0.473 \\
\hline Pelvic haematoma & 0 & $0.00 \%$ & 0 & $0.00 \%$ & 1 & $0.20 \%$ & 0.439 \\
\hline Haemorrhage & 1 & $0.30 \%$ & 1 & $0.30 \%$ & 1 & $0.20 \%$ & 0.975 \\
\hline
\end{tabular}
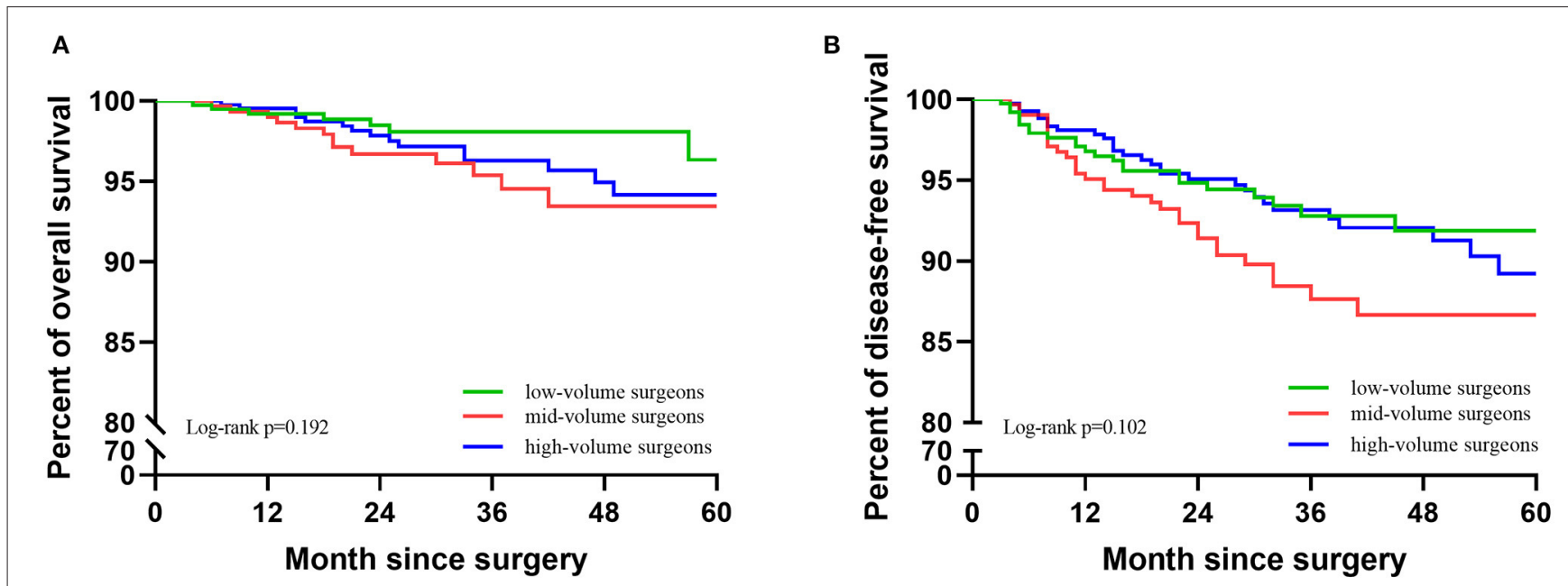

FIGURE 2 | Kaplan-Meier survival curves of the three groups. (A) KM of OS of the three groups. (B) KM of DFS of the three groups.

TABLE 3 | Association of surgical volume and survival in cervical cancer.

\begin{tabular}{|c|c|c|c|c|c|c|c|c|}
\hline \multirow[b]{2}{*}{ Project } & \multicolumn{3}{|c|}{ Disease-Free Survival } & \multicolumn{3}{|c|}{ Overall Survival } & \multirow{2}{*}{\multicolumn{2}{|c|}{$95 \% \mathrm{Cl}$}} \\
\hline & $P$ & HR & & $95 \% \mathrm{Cl}$ & $P$ & HR & & \\
\hline \multicolumn{9}{|c|}{ Before group matching } \\
\hline Low-volume group & 0.206 & & & & 0.239 & & & \\
\hline Mid-volume group & 0.084 & 2.515 & 0.884 & 7.157 & 0.126 & 1.596 & 0.877 & 2.902 \\
\hline High-volume group & 0.402 & 1.658 & 0.508 & 5.412 & 0.763 & 1.116 & 0.547 & 2.277 \\
\hline \multicolumn{9}{|c|}{ After group matching } \\
\hline Low- vs. Mid- & 0.051 & 4.526 & 0.994 & 20.622 & 0.120 & 1.800 & 0.859 & 3.775 \\
\hline Low- vs. High- & 0.839 & 11849135.200 & 0.000 & $1.438 \mathrm{E}+75$ & 0.426 & 2.018 & 0.359 & 11.354 \\
\hline Mid- vs. High- & 0.426 & 0.646 & 0.220 & 1.894 & 0.477 & 0.767 & 0.369 & 1.593 \\
\hline
\end{tabular}


TABLE 4 | Clinical and pathological characteristics among three groups after PSM matching.

\begin{tabular}{|c|c|c|c|c|c|c|c|c|c|c|c|c|c|c|c|}
\hline & \multicolumn{2}{|c|}{ Low } & \multicolumn{2}{|r|}{ Mid } & \multirow[t]{2}{*}{$P$} & \multirow{2}{*}{\multicolumn{2}{|c|}{$\begin{array}{c}\text { Low } \\
(n=145, \%)\end{array}$}} & \multicolumn{2}{|l|}{ High } & \multirow[t]{2}{*}{$\boldsymbol{P}$} & \multirow{2}{*}{\multicolumn{2}{|c|}{$\begin{array}{c}\text { Mid } \\
(n=202, \%)\end{array}$}} & \multirow{2}{*}{\multicolumn{2}{|c|}{$\begin{array}{c}\text { High } \\
(n=202, \%)\end{array}$}} & \multirow[t]{2}{*}{$\boldsymbol{P}$} \\
\hline & $(n=2$ & $001, \%)$ & & $(n=201, \%)$ & & & & $(n=145$ & & & & & & & \\
\hline Age (y) & 47.78 & \pm 9.524 & & $7.14 \pm 9.154$ & 0.345 & & $46.92 \pm 9.211$ & $47.21 \pm 9$ & 132 & 0.897 & 47.4 & $6 \pm 9.819$ & 47.3 & $33 \pm 9.241$ & 0.385 \\
\hline Hospital type & & & & & 0.916 & & & & & 0.602 & & & & & 0.773 \\
\hline General hospital & 123 & $61.20 \%$ & 119 & $59.20 \%$ & & 88 & $60.70 \%$ & 96 & $66.20 \%$ & & 119 & $58.90 \%$ & 112 & $55.40 \%$ & \\
\hline Tumor Hospital & 71 & $35.30 \%$ & 75 & $37.30 \%$ & & 52 & $35.90 \%$ & 44 & $30.30 \%$ & & 61 & $30.20 \%$ & 67 & $33.20 \%$ & \\
\hline Women-children specialty hospital & 7 & $3.50 \%$ & 7 & $3.50 \%$ & & 5 & $3.40 \%$ & 5 & $3.40 \%$ & & 22 & $10.90 \%$ & 23 & $11.40 \%$ & \\
\hline Region & & & & & 0.597 & & & & & 0.315 & & & & & 0.854 \\
\hline North China & 63 & $31.30 \%$ & 64 & $31.80 \%$ & & 71 & $49.00 \%$ & 73 & $50.30 \%$ & . & 81 & $40.10 \%$ & 84 & $41.60 \%$ & \\
\hline South China & 67 & $33.30 \%$ & 65 & $32.30 \%$ & & 36 & $24.80 \%$ & 23 & $15.90 \%$ & & 47 & $23.30 \%$ & 44 & $21.80 \%$ & \\
\hline Central China & 19 & $9.50 \%$ & 15 & $7.50 \%$ & & 18 & $12.40 \%$ & 21 & $14.50 \%$ & & 8 & $4.00 \%$ & 11 & $5.40 \%$ & \\
\hline East China & 47 & $23.40 \%$ & 46 & $22.90 \%$ & & 15 & $10.30 \%$ & 19 & $13.10 \%$ & & 34 & $16.80 \%$ & 28 & $13.90 \%$ & \\
\hline Southwest China & 5 & $2.50 \%$ & 11 & $5.50 \%$ & & 5 & $3.40 \%$ & 9 & $6.20 \%$ & & 32 & $15.80 \%$ & 35 & $17.30 \%$ & \\
\hline City class & & & & & 0.14 & & & & & 0.187 & & & & & 0.814 \\
\hline First-tier & 52 & $25.90 \%$ & 44 & $21.90 \%$ & & 33 & $22.80 \%$ & 21 & $14.50 \%$ & & 25 & $12.40 \%$ & 21 & $10.40 \%$ & \\
\hline Second-tier & 114 & $56.70 \%$ & 106 & $52.70 \%$ & & 84 & $57.90 \%$ & 91 & $62.80 \%$ & & 125 & $61.90 \%$ & 129 & $63.90 \%$ & \\
\hline Third-tier and above & 35 & $17.40 \%$ & 51 & $25.40 \%$ & & 28 & $19.30 \%$ & 33 & $22.80 \%$ & & 52 & $25.70 \%$ & 52 & $25.70 \%$ & \\
\hline Economics & & & & & 0.906 & & & & & 0.408 & & & & & 0.943 \\
\hline Countryside & 123 & $61.20 \%$ & 120 & $59.70 \%$ & & 93 & $64.10 \%$ & 90 & $62.10 \%$ & & 124 & $61.40 \%$ & 127 & $62.90 \%$ & \\
\hline Urban & 66 & $32.80 \%$ & 67 & $33.30 \%$ & & 44 & $30.30 \%$ & 41 & $28.30 \%$ & & 68 & $33.70 \%$ & 66 & $32.70 \%$ & \\
\hline Unknown & 12 & $6.00 \%$ & 14 & $7.00 \%$ & & 8 & $5.50 \%$ & 14 & $9.70 \%$ & & 10 & $5.00 \%$ & 9 & $4.50 \%$ & \\
\hline Year & & & & & 0.53 & & & & & 0.932 & & & & & 0.586 \\
\hline $2005-2013$ & 61 & $30.30 \%$ & 51 & $25.40 \%$ & & 47 & $32.40 \%$ & 46 & $31.70 \%$ & & 69 & $34.20 \%$ & 65 & $32.20 \%$ & \\
\hline 2014-2015 & 76 & $37.80 \%$ & 83 & $41.30 \%$ & & 51 & $35.20 \%$ & 54 & $37.20 \%$ & & 76 & $37.60 \%$ & 86 & $42.60 \%$ & \\
\hline 2016 & 64 & $31.80 \%$ & 67 & $33.30 \%$ & & 47 & $32.40 \%$ & 45 & $31.00 \%$ & & 57 & $28.20 \%$ & 51 & $25.20 \%$ & \\
\hline Q-M Type & & & & & 0.762 & & & & & 0.775 & & & & & 0.072 \\
\hline QM-B2 & 84 & $41.80 \%$ & 87 & $43.30 \%$ & & 32 & $22.10 \%$ & 30 & $20.70 \%$ & & 44 & $21.80 \%$ & 30 & $14.90 \%$ & \\
\hline QM-C2 & 117 & $58.20 \%$ & 114 & $56.70 \%$ & & 113 & $77.90 \%$ & 115 & $79.30 \%$ & & 158 & $78.20 \%$ & 172 & $85.10 \%$ & \\
\hline Number of lymph nodes removed & $18.67 \pm 9.765$ & $22.71 \pm 10.564$ & 0.454 & $21.49 \pm 10.808$ & $23.11 \pm 10.093$ & 0.697 & $22.48 \pm 10.347$ & $23.31 \pm 10.778$ & 0.808 & & & & & & \\
\hline Histology & & & & & 1 & & & & & 1 & & & & & 0.833 \\
\hline Squamous & 172 & $85.60 \%$ & 172 & $85.60 \%$ & & 130 & $89.70 \%$ & 130 & $89.70 \%$ & & 171 & $84.70 \%$ & 175 & $86.60 \%$ & \\
\hline Adenocarcinoma & 28 & $13.90 \%$ & 28 & $13.90 \%$ & & 14 & $9.70 \%$ & 14 & $9.70 \%$ & & 27 & $13.40 \%$ & 24 & $11.90 \%$ & \\
\hline Adenosquamous & 1 & $0.50 \%$ & 1 & $0.50 \%$ & & 1 & $0.70 \%$ & 1 & $0.70 \%$ & & 4 & $2.00 \%$ & 3 & $1.50 \%$ & \\
\hline
\end{tabular}


TABLE 4 | Continued

\begin{tabular}{|c|c|c|c|c|c|c|c|c|c|c|c|c|c|c|c|}
\hline & & ow & & & $P$ & & ow & & gh & $P$ & & id & & igh & $P$ \\
\hline & & $01, \%)$ & & $01, \%)$ & & & $145, \%)$ & & $45, \%)$ & & & $02, \%)$ & & $202, \%)$ & \\
\hline No & 175 & $87.10 \%$ & 172 & $85.60 \%$ & & 125 & $86.20 \%$ & 124 & $85.50 \%$ & & 168 & $83.20 \%$ & 166 & $82.20 \%$ & \\
\hline Yes & 26 & $12.90 \%$ & 29 & $14.40 \%$ & & 20 & $13.80 \%$ & 21 & $14.50 \%$ & & 34 & $16.80 \%$ & 36 & $17.80 \%$ & \\
\hline Deep stromal invasion & & & & & 0.821 & & & & & 0.521 & & & & & 0.896 \\
\hline No & 125 & $62.20 \%$ & 130 & $64.70 \%$ & & 101 & $69.70 \%$ & 92 & $63.40 \%$ & & 122 & $60.40 \%$ & 125 & $61.90 \%$ & \\
\hline Yes & 46 & $22.90 \%$ & 45 & $22.40 \%$ & & 30 & $20.70 \%$ & 35 & $24.10 \%$ & & 54 & $26.70 \%$ & 54 & $26.70 \%$ & \\
\hline Unreported & 30 & $14.90 \%$ & 26 & $12.90 \%$ & & 14 & $9.70 \%$ & 18 & $12.40 \%$ & & 26 & $12.90 \%$ & 23 & $11.40 \%$ & \\
\hline Uterine infiltration & & & & & 0.586 & & & & & 0.702 & & & & & 1 \\
\hline No & 195 & $97.00 \%$ & 193 & $96.00 \%$ & & 141 & $97.20 \%$ & 142 & $97.90 \%$ & & 193 & $95.50 \%$ & 193 & $95.50 \%$ & \\
\hline Yes & 6 & $3.00 \%$ & 8 & $4.00 \%$ & & 4 & $2.80 \%$ & 3 & $2.10 \%$ & & 9 & $4.50 \%$ & 9 & $4.50 \%$ & \\
\hline Parametrial involvement & & & & & 0.253 & & & & & 1 & & & & & 0.562 \\
\hline No & 199 & $99.00 \%$ & 196 & $97.50 \%$ & & 144 & $99.30 \%$ & 144 & $99.30 \%$ & & 200 & $99.00 \%$ & 201 & $99.50 \%$ & \\
\hline Yes & 2 & $1.00 \%$ & 5 & $2.50 \%$ & & 1 & $0.70 \%$ & 1 & $0.70 \%$ & & 2 & $1.00 \%$ & 1 & $0.50 \%$ & \\
\hline Surgical margin invasion & & & & & 0.562 & & & & & 0.316 & & & & & 0.317 \\
\hline No & 200 & $99.50 \%$ & 199 & $99.00 \%$ & & 145 & $100.00 \%$ & 144 & $99.30 \%$ & & 201 & $99.50 \%$ & 202 & $100.00 \%$ & \\
\hline Yes & 1 & $0.50 \%$ & 2 & $1.00 \%$ & & 0 & $0.00 \%$ & 1 & $0.70 \%$ & & 1 & $0.50 \%$ & 0 & $0.00 \%$ & \\
\hline Lymph node metastasis & & & & 0.874 & & & & & 0.527 & & & & & & 0.642 \\
\hline No & 178 & $88.60 \%$ & 179 & $89.10 \%$ & & 123 & $84.80 \%$ & 119 & $82.10 \%$ & & 180 & $89.10 \%$ & 177 & $87.60 \%$ & \\
\hline Yes & 23 & $11.40 \%$ & 22 & $10.90 \%$ & & 22 & $15.20 \%$ & 26 & $17.90 \%$ & & 22 & $10.90 \%$ & 25 & $12.40 \%$ & \\
\hline Adjuvant therapy & & & & 0.991 & & & & & 0.607 & & & & & & 0.645 \\
\hline None & 163 & $81.10 \%$ & 164 & $81.60 \%$ & & 115 & $79.30 \%$ & 108 & $74.50 \%$ & & 161 & $79.70 \%$ & 160 & $79.20 \%$ & \\
\hline Chemotherapy alone & 0 & $0.00 \%$ & 0 & $0.00 \%$ & & 0 & $0.00 \%$ & 0 & $0.00 \%$ & & 0 & $0.00 \%$ & 0 & $0.00 \%$ & \\
\hline Radiotherapy alone & 2 & $1.00 \%$ & 2 & $1.00 \%$ & & 2 & $1.40 \%$ & 2 & $1.40 \%$ & & 4 & $2.00 \%$ & 7 & $3.50 \%$ & \\
\hline Chemoradiotherapy & 36 & $17.90 \%$ & 35 & $17.40 \%$ & & 28 & $19.30 \%$ & 35 & $24.10 \%$ & & 37 & $18.30 \%$ & 35 & $17.30 \%$ & \\
\hline
\end{tabular}




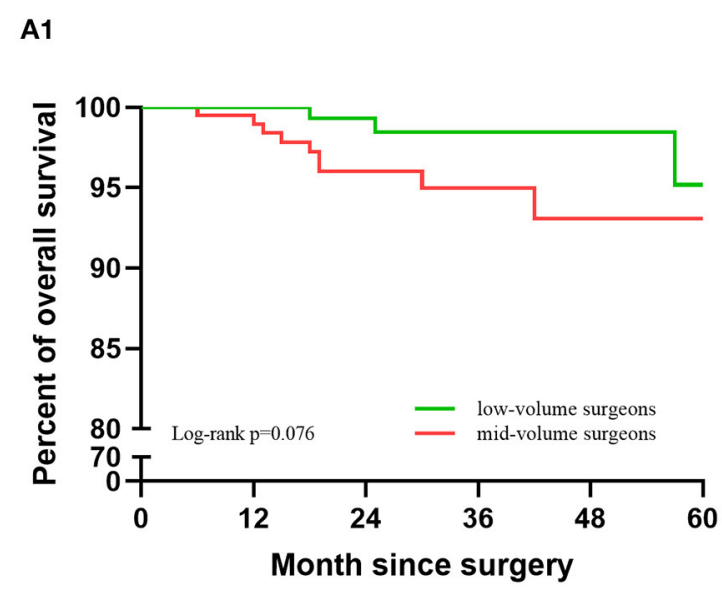

A2

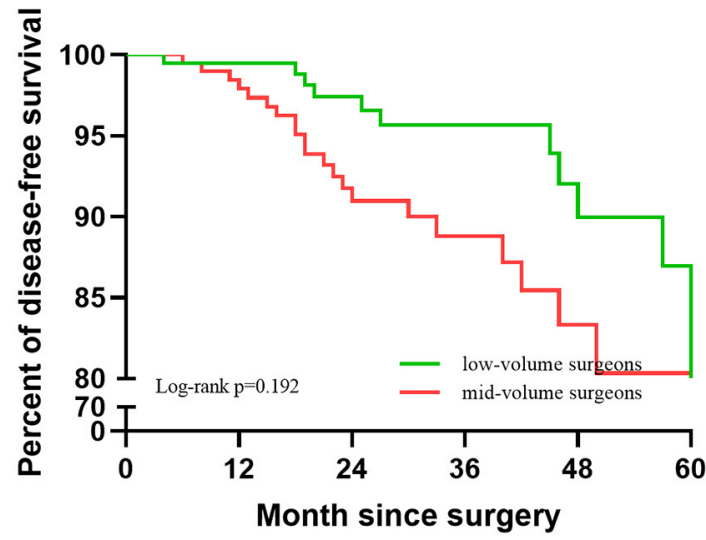

B2

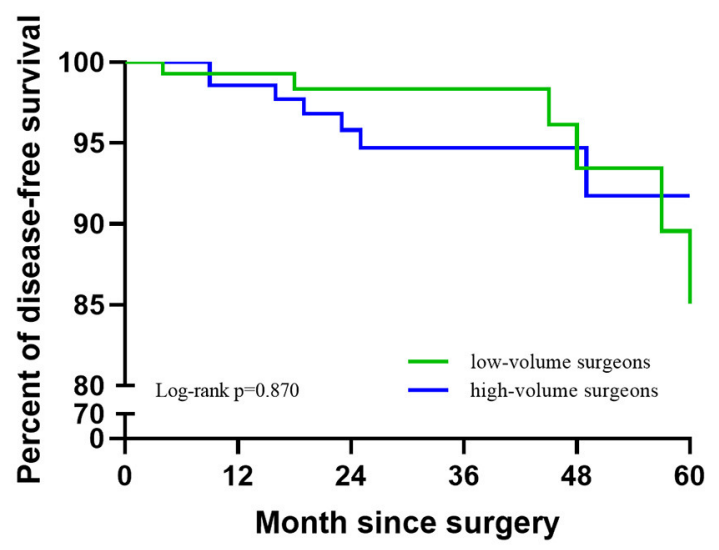

$\mathrm{C} 2$

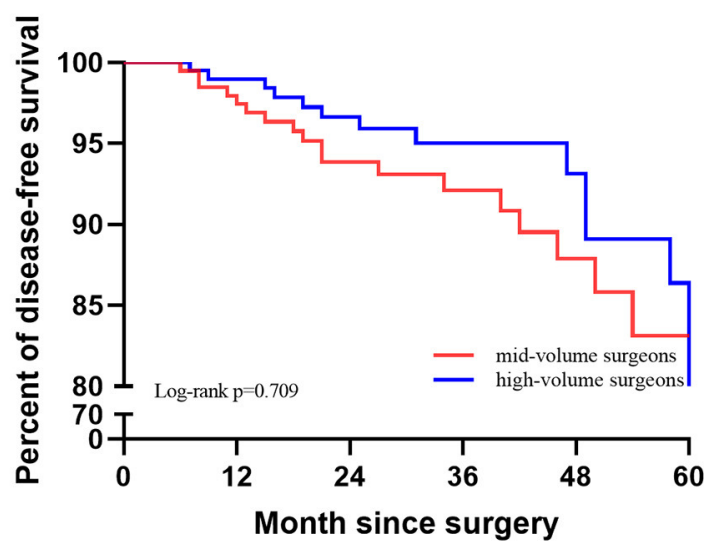

FIGURE 3 | Kaplan-Meier survival curves after PSM matching. (A) Low-volume group vs. mid-volume group. (B) Low-volume group vs. high-volume group. (C) Mid-volume group vs. high-volume group. 
relevant international guidelines, and these changes state that patients must be informed of the LACC results. Both NCCN (9) and European Society of Gynaecological Oncology (ESGO) $(25,26)$ stated that open surgery is the standard method for cervical cancer surgery. But these studies rarely analyzed the reasons of poor oncological outcomes of LRH.

Surgical experience may be one of the reasons influencing the oncology outcomes of LRH (13-15). But there are few studies analyzing the long-term survival impact of surgical experience in LRH. This large multicenter retrospective cohort study complements the evidence that surgical experience may not be a risk factor for LRH. The results of this study are similar to that of a recent retrospective study taken by scholar Chong et al. (13). They found similar 5-year OS and DFS between the first $50 \mathrm{LRH}$ patients and the second $50 \mathrm{LRH}$ patients performed by the same doctor and concluded that the increase in LRH experience may have no effect on oncological outcomes. They focused on the analysis of a single doctor, with good consistency, but the sample size was small. Our study was a multicenter, multisurgeon, large-sample analysis, and Cox regression analysis, and PSM were used to balance the case-mix factors; thus, the results are more convincing.

There are also studies with different results. In a multicenter study in Japan, Matsuo et al. (14) analyzed the oncological outcomes of 5,964 patients who underwent $\mathrm{RH}$ in 116 institutions and considered that high-volume hospitals may be associated with the risk of local recurrence and the improvement of survival rate. However, the surgical approaches were not included in the multivariable analysis because of the unavailable information. The surgical volume of institutions does not reflect the experience of surgeons. There may exist low-volume surgeons in highvolume institutions. Similarly, low-volume institutions may also have high-volume surgeons. This method may not exactly demonstrate how the surgical experience could affect the oncological outcomes. Our study makes up for the deficiency of Matsuo et al. We limited our inclusion criteria of patients who underwent LRH, and we focused on the specific experience of surgeons, which could better represent the effect of surgical experience on the efficacy of cervical cancer.

Laparoscopic surgery involves new technologies and equipment, with the surgical field being transformed from traditional three-dimensional open surgery to two-dimensional laparoscopic surgery. The increase in surgical experience means the repeated practice of surgical skills. In general, theoretical and practical research results confirm that laparoscopic surgery skills can be improved with an increase in surgical volume. Many previous learning curve studies of LRH have proved it (16-18). A meta-analysis to investigate the relationship between the number of gynecology surgeries and surgical outcomes has been conducted, and it is believed that intraoperative and postoperative complications are significantly reduced with high-volume surgeons (27). In the study of Chong et al. (13), it is undeniable that the operative time, hospital stay, time to restore normal residual urine volume, blood loss, intraoperative, and postoperative complications are significantly reduced and that the number of lymph nodes acquired is increased with an increase in the surgical experience. In our study, the surgical skills were significantly improved in the more experienced group, which is similar to Chong et al. Although the statistical difference is not significant among the three groups, the high-volume group had the trend of the higher incidence rate of complication. This may be because of the higher proportion of Q-M type C2 in the high-volume group.

However, we acknowledge several limitations in this study. First, this was a retrospective study with confounding factors. For example, in the high-volume group, the proportion of patients with LVSI and deep stromal invasion was higher than that in the mid- and low-volume groups. Nonetheless, we attempted to balance these differences through PSM. Second, the patient files and medical records among hospitals might be different, leading to the absence of clinical data, such as the information of the usage of a uterine manipulator, previous surgery, the surgical complexity score, or American Society of Anesthesiologists (ASA) score. Third, although our study covered a total of 46,313 cases of cervical cancer inpatients in 37 hospitals in most of China, it did not cover all regions nationwide. Fourth, we excluded cases that might affect the difficulty of surgery, such as the history of pelvic surgery, preoperative chemotherapy, or pelvic radiotherapy. However, anatomical variation, pelvic adhesion, and other parameters that may affect the difficulty of surgery are not available in our database. Fifth, some patients with endometrial cancer might receive $\mathrm{LRH}$, but this database was a cervical cancer-specialized disease database, so we did not consider the LRH volume of patients with endometrial cancer. Sixth, the ASA score of patients may influence the oncological outcomes, but the ASA score was not available in our database, so we did not consider the ASA score in this study.

Despite the above flaws, based on the large sample size of the study, we conclude the following clinical significance. First, we consider that surgical skills can be improved with rich experience. Shortening of the operative time and the reduction of intraoperative blood loss can minimize harm to patients during the operation. Second, surgical experience may not be the factor that affects the long-term oncological outcomes of LRH. Therefore, we need to further explore the limitations of laparoscopic technology itself.

\section{DATA AVAILABILITY STATEMENT}

The raw data supporting the conclusions of this article will be made available by the authors, without undue reservation.

\section{ETHICS STATEMENT}

The studies involving human participants were reviewed and approved by the Ethics Committee of Nanfang Hospital, Southern Medical University. The patients/participants provided their written informed consent to participate in this study.

\section{AUTHOR CONTRIBUTIONS}

CC, PL, LW, and JLa: study design. PL, JLi, and ZL: literature search. PL and JLi: figures. PL, CC, and XB: data analysis. PL and 
ZL: data collection. PL and JLi: drafting of the manuscript. CC: obtained funding. CC, LW, PL, and JLa: supervision. All authors data interpretation and approved the final version of the study.

\section{FUNDING}

This study was initially funded by the National Science and Technology Support Program of China (2014BAI05B03),

\section{REFERENCES}

1. Koh WJ, Greer BE, Abu-Rustum NR, Apte SM, Campos SM, Cho KR, et al. Cervical Cancer, 351 Version 2.2015. J Natl Compr Canc Netw. (2015) 13:395-404, 404. doi: 10.6004/jnccn.2015.0055

2. Nezhat CR, Burrell MO, Nezhat FR, Benigno BB, Welander CE. Laparoscopic radical hysterectomy with paraaortic and pelvic node dissection. Am J Obstet Gynecol. (1992) 166:864-5. doi: 10.1016/0002-9378(92) 91351-A

3. Vieira MA, Rendón GJ, Munsell M, Echeverri L, Frumovitz M, Schmeler KM, et al. Radical trachelectomy in early-stage cervical cancer: a comparison of laparotomy and minimally invasive surgery. Gynecol Oncol. (2015) 138:5859. doi: 10.1016/j.ygyno.2015.06.023

4. Wang YZ, Deng L, Xu HC, Zhang Y, Liang ZQ. Laparoscopy versus laparotomy for the management of early stage cervical cancer. BMC Cancer. (2015) 15:928. doi: 10.1186/s12885-015-1818-4

5. Bogani G, Cromi A, Uccella S, Serati M, Casarin J, Pinelli C, et al. Laparoscopic versus open abdominal management of cervical cancer: long-term results from a propensity-matched analysis. J Minim Invas Gyn. (2014) 21:85762. doi: 10.1016/j.jmig.2014.03.018

6. Hong JH, Choi JS, Lee JH, Eom JM, Ko JH, Bae JW, et al. Can laparoscopic radical hysterectomy be a standard surgical modality in stage IA2-IIA cervical cancer? Gynecol Oncol. (2012) 127:102-6. doi: 10.1016/j.ygyno.2012. 06.003

7. Diver E,Hinchcliff E,Gockley A, Melamed A, Contrino L, Feldman S, et al. Minimally invasive radical hysterectomy for cervical cancer is associated with reduced morbidity and similar survival outcomes compared with laparotomy. J Minim Invasive Gynecol. (2017) 24:402-6. doi: 10.1016/j.jmig.2016. 12.005

8. Ramirez PT, Frumovitz M, Pareja R, Lopez A, Vieira M, Ribeiro R, et al. Minimally invasive versus abdominal radical hysterectomy for cervical cancer. N Engl J Med. (2018) 379:1895-904 doi: 10.1056/NEJMoal80 6395

9. Koh WJ, Abu-Rustum NR, Bean S, Bradley K, Campos SM, Cho KR, et al. Cervical cancer, Version 3.2019, NCCN clinical practice guidelines in oncology. J Natl Compr Canc Netw. (2019) 17:64-84. doi: 10.6004/jnccn.2019.0001

10. Melamed A, Margul DJ, Chen L, Keating NL, Del Carmen MG, Yang J, et al. Survival after minimally invasive radical hysterectomy for early-stage cervical cancer. N Engl J Med. (2018) 379:1905-14. doi: 10.1056/NEJMoa180 4923

11. Doo DW, Kirkland CT, Griswold LH, McGwin G, Huh WK, Leath $\mathrm{CR}$, et al. Comparative outcomes between robotic and abdominal radical hysterectomy for IB1 cervical cancer: results from a single high volume institution. Gynecol Oncol. (2019) 153:242-7. doi: 10.1016/j.ygyno.2019. 03.001

12. Kim SI, Cho JH, Seol A, Kim YI, Lee M, Kim HS, et al. Comparison of survival outcomes between minimally invasive surgery and conventional open surgery for radical hysterectomy as primary treatment in patients with stage IB1-IIA2 cervical cancer. Gynecol Oncol. (2019) 153:3-12. doi: 10.1016/j.ygyno.2019.01.008

13. Chong GO, Park NY, Hong DG, Cho YL, Park IS, Lee YS. Learning curve of laparoscopic radical hysterectomy with pelvic and/or paraaortic lymphadenectomy in the early and locally advanced cervical cancer comparison of the first 50 and second 50 cases. Int $J$ Gynecol Cancer. (2009) 19:1459-64. doi: 10.1111/IGC.0b013e3181b7 6640 the National Natural Science Fund of Guangdong (2015A030311024), the Science and the Science and Technology Plan of Guangzhou (158100075), the Guangdong Medical Science and Technology Research Fund Project (A2020077), the Basic and Applied Basic Research Fund of Guangdong Province (2019A1515110337), the Chinese Postdoctoral Science Foundation (2019M660207), and the Nanfang Hospital President Fund (2019C005).

14. Matsuo K, Shimada M, Yamaguchi S, Matoda M, Nakanishi T, Kikkawa F, et al. Association of radical hysterectomy surgical volume and survival for early-stage cervical cancer. Obstet Gynecol. (2019) 133:1086-98. doi: 10.1097/AOG.0000000000003280

15. Vickers AJ, Savage CJ, Hruza M, Tuerk I, Koenig P, MartínezPiñeiro $\mathrm{L}$, et al. The surgical learning curve for laparoscopic radical prostatectomy: a retrospective cohort study. Lancet Oncol. (2009) 10:475-80. doi: 10.1016/S1470-2045(09)70079-8

16. Heo YJ, Kim S, Min KJ, Lee S, Hong JH, Lee JK, et al. The comparison of surgical outcomes and learning curves of radical hysterectomy by laparoscopy and robotic system for cervical cancer: an experience of a single surgeon. Obstet Gynecol Sci. (2018) 61:468-76. doi: 10.5468/ogs.2018.61. 4.468

17. $\mathrm{Li} \mathrm{XL}, \mathrm{Du} \mathrm{DF}$, Jiang $\mathrm{H}$. The learning curves of robotic and threedimensional laparoscopic surgery in cervical cancer. J Cancer. (2016) 7:23048. doi: $10.7150 /$ jca. 16653

18. Yim GW, Kim SW, Nam EJ, Kim S, Kim YT. Learning curve analysis of robot-assisted radical hysterectomy for cervical cancer: initial experience at a single institution. J Gynecol Oncol. (2013) 24:303-12. doi: 10.3802/jgo.2013.24. 4.303

19. Liu Y, Li L, Wu M, Ma S, Tan X, Zhong S, et al. The impact of the surgical routes and learning curve of radical hysterectomy on the survival outcomes in stage IB cervical cancer: a retrospective cohort study. Int J Surg. (2019) 68:72-7. doi: 10.1016/j.ijsu.2019.06.009

20. Hwang JH, Yoo HJ, Joo J, Kim S, Lim MC, Song YJ, et al. Learning curve analysis of laparoscopic radical hysterectomy and lymph node dissection in early cervical cancer. Eur J Obstet Gynecol Reprod Biol. (2012) 163:21923. doi: 10.1016/j.ejogrb.2012.05.005

21. Zhang W, Chen C, Liu P, Li W, Hao M, Zhao W, et al. Staging early cervical cancer in China: data from a multicenter collaborative. Int J Gynecol Cancer. (2019) 29:869-873. doi: 10.1136/ijgc-2019000263

22. Zhang $\mathrm{W}$, Chen $\mathrm{C}$, Liu $\mathrm{P}, \mathrm{Li} \mathrm{W}$, Hao $\mathrm{M}$, Zhao $\mathrm{W}$, et al. Impact of pelvic MRI in routine clinical practice on staging of IB1-IIA2 cervical cancer. Cancer Manag Res. (2019) 11:3603-9. doi: 10.2147/CMAR. S197496

23. Bhatla N, Aoki D, Sharma DN, Sankaranarayanan R. Cancer of the cervix uteri. Int J Gynaecol Obstet. (2018) 143(Suppl 2):22-36. doi: 10.1002/ijgo. 12611

24. Li Z, Chen C, Liu P, Duan H, Liu M, Xu Y, et al. Comparison of oncological outcomes and major complications between laparoscopic radical hysterectomy and abdominal radical hysterectomy for stage IB1 cervical cancer with a tumour size less than 2 cm. Eur J Surg Oncol. (2021) 47:212533. doi: 10.1016/j.ejso.2021.03.238

25. Querleu D, Cibula D, Concin N, Fagotti A, Ferrero A, Fotopoulou C, et al. Laparoscopic radical hysterectomy: a European Society of Gynaecological Oncology (ESGO) statement. Int J Gynecol Cancer. (2020) 30:15. doi: 10.1136/ijgc-2019-000775

26. Hillemanns P, Brucker S, Holthaus B, Kimmig R, Lampe B, Runnebaum I, et al. Updated opinion of the uterus commission of the gynecological oncology working group (AGO) and the gynecological endoscopy working group (AGE) of the German society of gynecology and obstetrics (DGGG) on the randomized study comparing minimally invasive with Abdominal Radical Hysterectomy for Early-stage Cervical Cancer (LACC). Geburtshilfe Frauenheilkd. (2019) 79:145-7. doi: 10.1055/a-08247929

27. Mowat A, Maher C, Ballard E. Surgical outcomes for lowvolume vs high-volume surgeons in gynecology surgery: a 
systematic review and meta-analysis. Am J Obstet Gynecol. (2016) 215:21-33. doi: 10.1016/j.ajog.2016.02.048

Conflict of Interest: The authors declare that the research was conducted in the absence of any commercial or financial relationships that could be construed as a potential conflict of interest.

Publisher's Note: All claims expressed in this article are solely those of the authors and do not necessarily represent those of their affiliated organizations, or those of the publisher, the editors and the reviewers. Any product that may be evaluated in this article, or claim that may be made by its manufacturer, is not guaranteed or endorsed by the publisher.

Copyright (C) 2021 Li, Liu, Wang, Kang, Yang, Guo, Yao, Lu, Wang, Lin, Li, Bin, Lang, Liu and Chen. This is an open-access article distributed under the terms of the Creative Commons Attribution License (CC BY). The use, distribution or reproduction in other forums is permitted, provided the original author(s) and the copyright owner(s) are credited and that the original publication in this journal is cited, in accordance with accepted academic practice. No use, distribution or reproduction is permitted which does not comply with these terms. 